\title{
I-SSR Markers Revealed Inconsistent Phylogeographic Patterns among Populations of Japanese Red Pines in Korea
}

\author{
By Y.-P. HoNG ${ }^{1,3)}$, H.-Y. KwoN ${ }^{1)}$, I.-S. KIM ${ }^{2)}$
}

(Received $21^{\text {st }}$ September 2005)

\begin{abstract}
To monitor the level and distribution pattern of genetic diversity in countrywide populations of Japanese red pines in Korea, 80 I-SSR variants were analyzed from 192 individuals in 11 populations. The previously reported data, obtained from the 8 populations of this species, were incorporated into the estimation of the population genetic statistics. Relatively higher level of genetic diversity was observed in 19 populations of Japanese red pines (mean of 0.453) than those in other tree species. From the results of AMOVA, majority of genetic diversity $(92 \%)$ was allocated within populations, which brought about the moderate degree of population differentiation $\left(\Phi_{\mathrm{ST}}=0.08\right)$. Two genetic groups were observed from the dendrogram reconstructed by UPGMA. Overall correlation between genetic relationships and geographic affinity was inconsistent among the 19 populations. Some probable genetic disturbances, induced by either human interference or the natural process of regeneration for the species after devastation, might be responsible for the discrepancy between genetic relationships and geographical distribution of the populations. These results suggested that simple geographic or ecological grouping might not represent the genetic zone of the Korean populations of Japanese red pines, and that more careful approach should be made for designating in situ gene conservation of this species in Korea.
\end{abstract}

Key words: gene conservation, genetic diversity, genetic relationships, I-SSR variants, AMOVA, population differentiation.

\section{Introduction}

Japanese red pines (Pinus densiflora Sieb. et Zucc.) is one of the most important conifers in terms of both economy and culture, and also holds an important position in forest ecosystem in Korea. It frequently occurs naturally as pure stands in diverse sites of various soil and climatic conditions (LEE, 1987). It had been extensively used as materials for building construction, shipbuilding, furniture, coffins, firewood, and different valuable by-products (LEE, 1987; FRI, 1999). The high demand for the wood of this species had resulted in devastation of the forests of Japanese red pines in Korea. According to the historical records on Chosun Dynasty (1392-1910 A.D.), there were several records on the intensive logging and the royal efforts for the protection and reforestation of this pine. For example, logging without per-

1) Dept. of Forest Genetic Resources, Korea Forest Research Institute, Suwon 441-350, Republic of Korea.

$\left.{ }^{2}\right)$ Forest Seed Research Center, Chungju, 380-940, Republic of Korea.

3) To whom correspondence should be addressed. Phone: 082-031290-1142. Fax: 082-031-290-1040. E-mail: yphong@foa.go.kr mission was prohibited by the royal edict and reforestation of them was performed in ever place of the country (reviewed in FRI, 1999). However, until the mid $20^{\text {th }}$ century, a large portion of the forests were devastated by being cut down for firewood and/or being burnt out by war fire through the Korean War (1950-1953), which resulted in the large area of naked surface on mountains. Such naked forestlands might provide good chance for natural regeneration of the Japanese red pines that is known as leading species occupying the open forestland. This might also result in random changes in genetic composition of the regenerated populations of Japanese red pines in Korea (i.e., founder effects). Since the early 1960s, Korean government has made tremendous efforts to reforest nearly naked mountain area. As a result, forests of Japanese red pines have been retrieved their former positions now. Accordingly, for the more effective uses and sustainable management of genetic resources of the Japanese red pines in the future, we should make an effort to understand population genetic architecture of the current forests by broadening the knowledge on the level and distribution of genetic diversity.

In the present paper, the level and distribution of ISSR variants were estimated in the eleven populations of Japanese red pines in Korea, which were also compared with the other previous results obtained by analyzing other genetic marker systems. This study might provide valuable information for the establishment of efficient conservation practices on Japanese red pines in Korea.

\section{Materials and Methods \\ Plant Material \& DNA Extraction}

Open-pollinated seeds were collected from 192 individual trees in 11 populations located throughout the native range of Japanese red pines in South Korea (Fig. 1). Within each population, 14-19 trees were selected for seed collection with a minimum distance of $30 \mathrm{~m}$ in order to avoid selecting genetically closely related ones. Individual total DNAs were extracted by the modified CTAB method from the respective pooled samples of 10 megagametophytes to cover all allelic status in whole loci (Hong et al., 1993). After agarose gel electrophoresis, the amount of DNA was indirectly quantified by comparing band intensity with known quantity standard of uncut DNA.

\section{PCR Amplification \& Electrophoresis}

Polymerase chain reactions (PCR) were carried out in a volume of $20 \mu \mathrm{l}$ with final concentration of $10 \mathrm{ng}$ of 


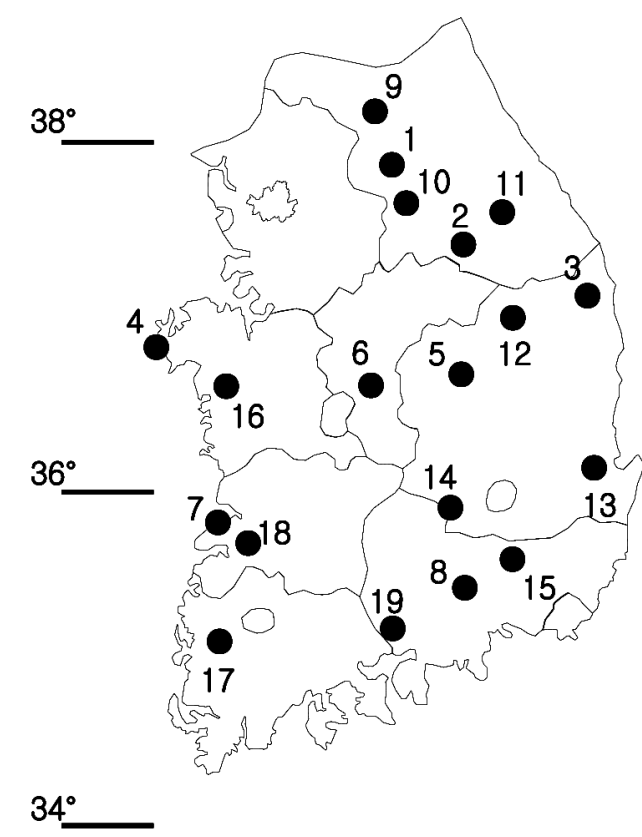

Figure 1. - Location of sampled populations. Population names refer to those corresponding to the numbers in Table 1.

template DNA; 1x PCR reaction buffer, $1.75 \mathrm{mM}$ of $\mathrm{MgCl}_{2}, 0.2 \mathrm{mM}$ of dNTPs, $0.0025 \%$ BSA, 0.6 unit of Taq DNA polymerase (Advanced Biotechnologies Ltd., UK) and $0.4 \mu \mathrm{M}$ of I-SSR primer. Thermal cycling were performed in a Primus $96^{\text {plus }}$ thermal cycler (MWG AG Biotech, Germany) as follows; a single cycle of $5 \mathrm{~min}$ of initial denaturation at $94^{\circ} \mathrm{C}$, followed by 45 cycles of 30 sec of denaturation at $94^{\circ} \mathrm{C}, 30 \mathrm{sec}$ of annealing at $52^{\circ} \mathrm{C}$, 1 min of extension at $72^{\circ} \mathrm{C}$, and a final extension step of $10 \mathrm{~min}$ at $72^{\circ} \mathrm{C}$. After amplification, PCR products were fractionated by using $2 \%(\mathrm{w} / \mathrm{v})$ agarose gels containing ethidium bromide with a $1 \mathrm{x}$ TBE buffer at $\mathrm{pH} 8.0$ for $2.5 \mathrm{~h}$ and then photographed over UV trans-illuminator. To insure consistency of PCR procedure, semi-automation of I-SSR PCR analysis - including distribution of template DNA, PCR mixture and loading buffer, and automatic gel loading - was attained by using RoboSeq ${ }^{\circledR}$ 4204S Automated Biosystem (MWG AG Biotech, Germany).

\section{Data Analysis}

The observed I-SSR variants were recorded as the presence (1) versus the absence (0) of the same size. In order to monitor the countrywide pattern of genetic diversity and genetic relationship among populations, additional data from the 8 populations, analyzed in the previous study (HoNG et al., 2004), were incorporated to the following statistical analysis. To estimate the distribution of I-SSR variants among the categories of presence or absence within population, the Shannon's index (S.I.; Shannon, 1948) was calculated using the POPGENE 1.31 program (YEH et al., 1999). Level of genetic differentiation among populations was estimated at 2 and 3 hierarchical levels by AMOVA on the basis of genetic distance calculated by Euclidean metric of EXCOFFIER et al. (1992) using Arlequin 2.0 program (SchneIDER et al., 2000). Genetic relationships among populations were reconstructed by UPGMA (phylip v3.5c; FELSENSTEIN, 1993) on the basis of pair-wise Manhattan distance (WRIGHT, 1978) between populations that was computed by RAPDDIST v1.0 (BLACK, 1996). Statistical test for the topology of each node was performed with 100 bootstrapped samples prepared by RAPDDIST v1.0 (BLACK, 1996).

\section{Results}

PCR was performed with 6 I-SSR primers that were used in the previous study with 8 populations (Hong et al., 2004). A total of 80 I-SSR variants, which were scored in the previous study (HoNG et al., 2004), were considered to be the subject of this study with 11 populations of Japanese red pines [UBC \#809(12 variants), 811(11), 818(14), 826(17), 846(16), 873(10)]. On the basis of pooled phenotypic data of the observed 80 I-SSR variants, none of the analyzed individuals showed identical profiles. Relatively high level of genetic diversity was observed within 19 populations, ranged from 0.411 (Gyeongbuk-Mungyeong) to 0.505 (GyeongbukGoryeong) with the mean of 0.453 (Table 1), compared to other tree species - i.e. Torreya nucifera: 0.353 (HoNG et al., 2000), Ginkgo biloba: 0.379 (HonG et al., 2001), Rubus coreanus: 0.242 (HoNG et al., 2003), Vaccinium uliginosum: 0.47 (HAN et al., 2005).

A result of AMOVA with 19 populations revealed that majority of genetic diversity was allocated within populations $(92 \%)$, which resulted in moderate degree of genetic differentiation among populations $\left(\Phi_{\mathrm{ST}}=0.08\right.$, Table 2). When AMOVA was performed at 3 hierarchical levels on the basis of the results of analysis of genetic relationship among 19 populations, where 19 populations could be grouped as 2 genetic groups, proportion of genetic differentiation estimated among total populations $\left(\Phi_{\mathrm{ST}}=0114\right)$ was near evenly distributed both between 2 genetic groups $\left(\Phi_{\mathrm{SC}}=0.056\right)$ and among populations within groups $\left(\Phi_{\mathrm{CT}}=0.061\right)$. This observation suggested that voluntary grouping on the basis of genetic relationships was also genetically meaningful. Observed degree of population differentiation in Japanese red pines $\left(\Phi_{\mathrm{ST}}=0.08\right)$ was higher than that observed in Ginkgo biloba (0.057; HoNG et al., 2001) and lower than those of Torreya nucifera (0.094; HoNG et al., 2000), Rubus coreanus (0.185, Hong et al., 2003), and Vaccinium uliginosum (0.335, HAN et al., 2005).

Genetic relationships among 19 populations were reconstructed by UPGMA (Fig. 2), which revealed 2 genetic groups. Two populations of ChungnamCheongyang and Jeonbuk-Jeongeup revealed the closest genetic relationship with the confidence interval of $81 \%$ that was obtained from the replicated analyses with the bootstrapped 100 pseudo-replicate data sets. Even though they are not the most closely located populations, they showed the closest genetic relationship that represented the largest proportion of genetic variation being shared between them. This observation suggested that there might be high probability of sharing the same hypothetical progenitor for both populations and undergone similar evolutionary process. Theoretically, if natural populations were originated from the same hypo- 
Table 1. - Diversity of I-SSR variants within population.

\begin{tabular}{lccc}
\hline \multicolumn{1}{c}{ Population } & Individuals & S.I. & Citation \\
\hline 1. Gangwon-Hongcheon & 17 & 0.421 & Hong et al. 2004 \\
2. Gangwon-Yeongwol & 19 & 0.472 & Hong et al. 2004 \\
3. Gyeongbuk-Uljin & 19 & 0.442 & Hong et al. 2004 \\
4. Chungnam-Taean & 20 & 0.451 & Hong et al. 2004 \\
5. Gyeongbuk-Mungyeong & 20 & 0.411 & Hong et al. 2004 \\
6. Chungbuk-Boeun & 18 & 0.428 & Hong et al. 2004 \\
7. Jeonbuk-Buan & 19 & 0.495 & Hong et al. 2004 \\
8. Gyeongnam-Haman & 18 & 0.483 & Hong et al. 2004 \\
9. Gangwon-Hwacheon & 17 & 0.397 & \\
10. Gangwon-Hoengseong & 18 & 0.461 & \\
11. Gangwon-Jeongseon & 19 & 0.465 & \\
12. Gyeongbuk-Bonghwa & 15 & 0.432 & \\
13. Gyeongbuk-Pohang & 19 & 0.437 & \\
14. Gyeongbuk-Goryeong & 19 & 0.505 & \\
15. Gyeongnam-Milyang & 18 & 0.491 & \\
16. Chungnam-Cheongyang & 19 & 0.460 & \\
17. Jeonnam-Naju & 16 & 0.468 & \\
18. Jeonbuk-Jeongeup & 14 & 0.446 & \\
19. Gyeongnam-Hadong & 18 & 0.437 & \\
\hline \multicolumn{1}{c}{ Average } & 18 & 0.453 & \\
\hline
\end{tabular}

Table 2. - Results of AMOVA for 19 populations. Groups were voluntarily designated on the basis of genetic relationship among populations observed in this study.

\begin{tabular}{ccc}
\hline Source of variance & d.f. & Component Variance \\
\hline Among populations & 18 & $8.0 \%$ \\
Within populations & 324 & $92.0 \%$ \\
\hline Among groups & 1 & $6.12 \%$ \\
Among populations within groups & 17 & $5.29 \%$ \\
Within populations & 322 & $88.59 \%$ \\
\hline
\end{tabular}

$\phi_{\mathrm{ST}}=0.114: \Phi_{\mathrm{SC}}=0.056: \Phi_{\mathrm{CT}}=0.061$

$\phi_{\mathrm{ST}}$ : genetic differentiation among populations

$\phi_{\mathrm{SC}}$ : genetic differentiation among populations within groups

$\phi_{\mathrm{CT}}$ : genetic differentiation between 2 genetic groups

thetical progenitor and undergone stable evolutionary process, geographically closer populations should show closer genetic relationship. Although 3 geographically adjacent populations of Gangwon (i.e., Hongcheon, Hwacheon, and Hoengseong) showed the closest genetic relationships (C.I. $=84 \%$ ), overall genetic relationships among the 19 populations were not coincided with geographic affinity. For example, in addition to the example of a population pair of Chungnam-Cheongyang and Jeonbuk-Jeongeup, one of the genetically close population pairs, Gyeongbuk-Ulgin and Chungnam-Taean (C.I. $=73 \%$ ), was also located quite far from each other (Fig. 1). Furthermore, the most closely located population of Jeonbuk-Buan to that of Jeonbuk-Jeongeup was grouped into different genetic group, and meanwhile showed the closest genetic relationship with the far located population of Gyeongbuk-Goryeong.

\section{Discussion}

The distribution of I-SSR variants among populations of Japanese red pines showed typical patterns that were seen in long-lived woody species. In general, long-lived woody species usually show less than $10 \%$ of genetic differentiation among populations (HAMRICK et al., 1992). Although the distribution pattern of genetic diversity among populations of Japanese red pines was turned out to be typical for the long-lived woody species, level of population differentiation observed in this study was much lower than that observed in the earlier studies estimated on the basis of I-SSR marker $\left(\Phi_{\mathrm{ST}}=0.135\right.$, KIM et al., 2003) and RAPD marker analyses $\left(\mathrm{G}_{\mathrm{ST}}=0.129\right.$, LEE et al., 1997) for the same species of different populations in Korea.

For two independent studies with I-SSR marker analyses, it might be unrealistic to compare the results 


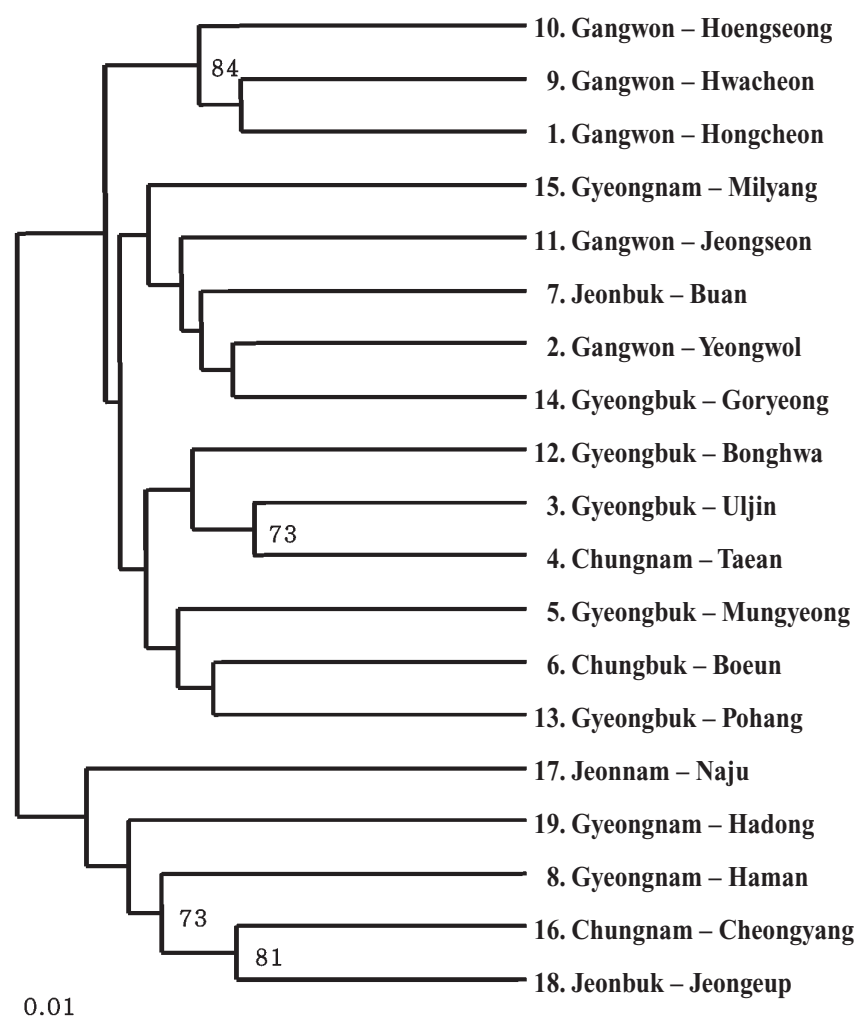

$\underline{0.01}$

Figure 2. - Dendrogram reconstructed by UPGMA. Figures at the node represented confidence intervals estimated from 100 bootstrapping. Only confidence intervals of higher than $50 \%$ were indicated.

obtained from such different data sets directly because different entries of populations and I-SSR markers were included. Nevertheless, the fact that the estimation of degree of population differentiation observed in this study $\left(\Phi_{\mathrm{ST}}=0.08\right)$ was only equivalent to three fifth of that observed in the other study $\left(\Phi_{\mathrm{ST}}=0.135\right.$; KIM et al., 2003) was noticeable. Interestingly, although more number of individuals from each population was analyzed in the other study (average of 29; KIM et al., 2003) compared to the present study (average of 18.75), the observed genetic diversity within populations was much lower in the other study (mean of 0.392 ) compared to the present study (mean of 0.453). There might be several probable causes for such difference in the level of observed genetic diversity. First, sampling difference in selecting individual samples and/or I-SSR markers for the independent analyses should not be overlooked. Second, at least the 2 populations showing the lowest genetic diversity [i.e., Yesan (0.287) and Iksan (0.3); KIM et al., 2003] and the closest genetic relationship (refer to Fig. 3 in the paper of KIM et al., 2003) in the earlier study might reflect that they might be undergone recent population shrinkage caused by severe disturbances such as logging, war fire during the Korean War, and artificial fire for preparing forest ground for cultivation. Such abrupt changes in population size might also result in lessening of genetic variation (HARTL and CLARK, 1989). Several archival evidences for such postulation could be found in Pibyonsa Tungnok (NIKH, 2001) and a True Record of the Chosun Dynasty (NIKH, 1999). From the old days, Japanese red pines have grown near to human residence and extensively used as materials for building construction, shipbuilding, furniture, coffins, firewood, and different valuable by-products (LEE, 1987; FRI, 1999). Therefore, although exact names of the places were not notified, several records about giving warning against severe logging of Japanese red pines were found in the archives prepared during the $14^{\text {th }}$ century $(\mathrm{NIKH}, 1999)$ and the $17^{\text {th }}$ century (NIKH, 2001).

Although it is not realistic to compare the results obtained from different marker systems and different statistics (i.e., $\Phi_{\mathrm{ST}}$ for I-SSR markers in the present study vs. $\mathrm{G}_{\mathrm{ST}}$ for RAPD markers in the earlier study, LEE et al., 1997) directly, much higher degree of population differentiation was observed for RAPD markers $\left(\mathrm{G}_{\mathrm{ST}}=0.129\right)$ than I-SSR markers $\left(\Phi_{\mathrm{ST}}=0.09\right)$. Different nature of RAPD and I-SSR variants representing different target regions of the genome might be partially responsible for such difference in the degree of population differentiation (WILliams et al., 1990; SAGHAI et al., 1994; SAlimath et al., 1995; Tsumura et al., 1996). The fact, that different target regions of the genome were analyzed by PCR using either I-SSR or RAPD primers, raises a conjecture that they might be undergone different evolutionary processes (ex. different rates of nucleotide mutation for different priming sites: arbitrary 10 nucleotides of high $\mathrm{G}+\mathrm{C}$ content for RAPD vs. a few repeats of the simple sequences with 1 to 3 additional arbitrary anchor nucleotides for I-SSR analyses). In addition to the above-mentioned possible reasons, in the earlier study (LEE et al., 1997), RAPD analysis was performed only with the Mendelian loci where RAPD variants showed a 1:1 segregation ratio. By analyzing those markers showing a 1:1 segregation, they calculated allelic frequencies from the observed dominant phenotypes under the assumption of all populations being reached at Hardy-Weinberg equilibrium. This might result in overestimation of the degree of population differentiation on the basis of only 30 Mendelian RAPD loci.

The discrepancy between geographic distribution and genetic relationships among the analyzed populations suggested that they might be undergone random changes in genetic composition, resulted from some kinds of disturbances, such as logging, war fire, artificial fire for preparing forest ground for cultivation, and extensive afforestation etc. Random genetic drift induced by drastic changes in population size and transplantation from other populations for reforestation (FRI, 1999) might be responsible for such discrepancy between geographic distance and genetic relationship. Grouping of the geographically distant populations of Chungnam-Cheongyang and Jeonbuk-Jeongeup with relatively high confidence interval $(81 \%)$ might not be simply explained by population differentiation via the process of stable population expansion and isolation. There might be several probable processes to result in such phenomenon that could be either relatively recent fractionation of the panmictic population, fortuitous sampling during natural regeneration after disturbance, or recent transplantation from either one of them.

Observation on the distribution of I-SSR variants, degree of genetic differentiation, and genetic relation- 
ships among the populations of Japanese red pines in Korea suggested that information on genetic zoning should be considered more carefully during preparing the strategy for gene conservation of Japanese red pines in Korea. By considering genetic zones for in situ gene conservation, proper selection of candidate populations for conservation could be secured. For example, although some populations (ex. 2 population pairs of Chungnam-Cheongyang/Jeonbuk-Jeongeup and Gyeongbuk-Uljin/Chungnam-Taean) are located far from each other, selection of only one from each group for in situ genetic conservation might be good enough for securing genetic diversity in both groups. This also prevents probable mistake of selecting improper candidate populations by making decision only on the basis of geographic zone or provenance regions for gene conservation (CHOI et al., 1999). In conclusion, in order to prepare more reasonable strategy for gene conservation of Japanese red pines in Korea, molecular population genetic information on the level and distribution pattern of genetic diversity in other genomes of the same individuals, such as chloroplast DNA and mitochondria DNA, should be incorporated in future.

\section{Literature}

BLACK, W. C. IV. (1996): RAPDDIST 1.0. Department of Microbiology, Colorado State University, Fort Collins, CO. USA.

CHOI, W.-Y., W.-S. TAK, K.-B. YIM and S.-S. JANG (1999): Delineation of provenance regions of forests based on climate factors in Korea. Jour. Korean For. Soc. 88(3): 379-388 (in Korean).

Excoffire, L., P. Smouse and J. QuatTro (1992): Analysis of molecular variance inferred from metric distance among DNA haplotypes: application to human mitochondrial DNA restriction data. Genetics 131: 479-491.

Felsenstein, J. (1993): PHYLIP (Phylogeny Inference Package) version 3.5c. Distributed by the author. Dept. of Genetics, Univ. of Washington, Seattle, WA, USA.

Forest Research Institute (FRI) (1999): Pine, Pine Forest. Seoul, Korea (in Korean).

Hamrick, J. L., M. J. W. Godt and S. L. Sherman-Broyles (1992): Factors influencing levels of genetic diversity in woody plant species. New Forests 6: 95-124.

HartL, D. L. and A. G. Clark (1989): Principles of population genetics. $2^{\text {nd }}$ edition. Sinauer Associates, Sunderland, MA, USA pp.282-296.

HaN, S.-D., Y.-P. Hong, H.-Y. Kwon, B.-H. YANG and C.-S. KIM (2005): Genetic variation of 2 isolated populations of Vaccinium uliginosum L. in Korea. J. Kor. for. Soc. (in press).

Hong, Y.-P., V. D. Hipkins and S. H. STRAuss (1993): Chloroplast DNA diversity among trees, populations and species in the California closed-cone pines (Pinus radiata, $P$. muricata and $P$. attenuata). Genetics 135: 1187-1196.

Hong, Y.-P., K.-J. CHO and E.-M. SHIN (2000): Diversity of I-SSR variants in the populations of Torreya nucifera. J. Kor. for. Soc. 89(2): 167-172.

Hong, Y.-P., K.-J. CHO, K.-N. HoNG and E.-M. SHIN (2001): Diversity of I-SSR variants in Ginkgo biloba L. planted in 6 regions of Korea. J. Kor. for. Soc. 90(2): 169-175.
HonG, Y.-P., M.-J. KIM and K.-N. HoNG (2003): Genetic diversity in natural populations of two geographic isolates of Korean black raspberry. J. Hort. Sci. Biotech. 78(3): 350-354.

HonG, Y.-P., H.-Y. Kwon, K.-S. KIM, K.-N. Hong and Y.-Y. KIM (2004): Discordance between geographical distribution and genetic relationship among populations of Japanese red pines in Korea revealed by analysis of ISSR markers. Silvae Genetica 53(3): 89-92.

Kim, Z.-S., R. WANG, P. G. Gorovoy and H.-Y. KWON (2003): Distribution of I-SSR and mtDNA Variants in 19 Populations of Pinus densiflora in Korea, China and Russia. J. Kor. for. Soc. 93(2): 152-159.

LEE, S.-W., Y.-Y. KIM, J.-O. HYUN and Z.-S. KIM (1997): Comparison of genetic variation in Pinus densiflora natural populations by allozyme and RAPD analysis. Kor. J. Breed. 29(1): 72-83 (in Korean).

LEE, T.-B. (1987): Dendrology. Hyang Moon Sa, Seoul, Korea (in Korean).

National Institute of Korean History (NIKH) (1999): Chosun Dynasty Silok. Seoul, Korea (in Korean).

NATIONAL INSTITUTE OF KOREAN History (NIKH) (2001): Pibyonsa Tungnok. Seoul, Korea (in Korean).

PARK, P.-S. and D.-G. LEE (1996): Factors affecting the natural regeneration of Pinus densiflora $\mathrm{S}$. et $\mathrm{Z}$. after forest works at the Mt. Joongwang located in Pyungchang-gun, Kangwon-do. Proceedings of Academic Conference of Kor. For. Soc. pp. 57-58 (in Korean).

Saghai, M. A., R. M. Biyashev, G. P. Yang, Q. Zhang and R. W. AlLARD (1994): Extraordinarily polymorphic microsatellite DNA in barley: species diversity, chromosomal locations, and population dynamics. Proc. Natl. Acad. Sci. USA 91: 5466-5470.

Salimath, S. S., A. C. De-Oliveira, I. D. Godwin and J. L. BENNETZEN (1995): Assessment of genome origins and genetic diversity in the genus Eleusine with DNA markers. Genome 38(4): 757-763.

ShanNon, C. E. (1948): A mathematical theory of communication. Bell System Tech. J. 27: 379-423.

Schneider, S., D. Roessli and L. Excoffier (2000): Arlequin v.2.0. A software for population genetics data analysis. Genetics and biometry laboratory, Dept. of anthropology and ecology, Univ. of Geneva, Switzerland.

Tsumura, Y., K. OHBA and S. H. Strauss (1996): Diversity and inheritance of inter-simple sequence repeat polymorphisms in Douglas-fir (Pseudotsuga menziesii) and sugi (Cryptomeria japonica). Theor. Appl. Genet. 92: 40-45.

Williams, J. G. K., A. R. KubELIK, K. J. LivaK, J. A. RAFALSKI and S. V. TINGEY (1990): DNA polymorphisms amplified by arbitrary primers are useful as genetic markers. Nucleic Acids Res. 18: 6531-6535.

WRIGHT, S. (1978): Evolution and genetics of populations. Vol. 4. Variability within and among natural populations. Univ. of Chicago Press, Chicago, USA.

YEH, F. C., R. C. YANG and T. BoYLe (1999): POPGENE v 1.31. Microsoft window-based freeware for population genetic analysis. Dept. of Renewable Resources. Univ. of Chicago Press, Chicago, USA.

YIM, K.-B. (1962): Analytical studies on Pinus densiflora stand development in the middle part of Korea. Res. Bull. of Kor. Agric. Soc. 39-47.

YIM, K.-B. (1996): Principles of Silviculture. Hyang Moon Sa, Seoul, Korea (in Korean). 